\title{
Enantioselective Synthesis of Apoptolidinone: Exploiting the Versatility of Thiazolidinethione Chiral Auxiliaries
}

Michael T. Crimmins, ${ }^{*}$ Hamish S. Christie, Kleem Chaudhary and Alan Long

Department of Chemistry, Venable and Kenan Laboratories of Chemistry, University of North Carolina at Chapel Hill, Chapel Hill, North Carolina 27599-3290

Supporting Information: Experimental Procedures 


\section{Experimental Procedures}

Proton and carbon nuclear magnetic resonance spectra were recorded at either 500 or 400 $\mathrm{MHz}\left({ }^{1} \mathrm{H}\right)$ and 125 or $100 \mathrm{MHz}\left({ }^{13} \mathrm{C}\right)$. Chemical shifts are reported in ppm with residual undeuterated solvent peaks as internal reference for ${ }^{1} \mathrm{H}$ NMR: $\mathrm{CHCl}_{3}(7.26), \mathrm{C}_{6} \mathrm{D}_{6}(7.15)$, $\mathrm{CD}_{3} \mathrm{OD}$ (3.31) and deuterated solvent shifts for ${ }^{13} \mathrm{C} \mathrm{NMR} \mathrm{CHCl}_{3}$ (77.0), $\mathrm{C}_{6} \mathrm{D}_{6}$ (128.06), $\mathrm{CD}_{3} \mathrm{OD}$ (49.00). ${ }^{1}$ Multiplicities are reported as (s) singlet, (d) doublet, (t) triplet, (q) quartet and $(\mathrm{m})$ multiplet. Unresolved, overlapping resonances are reported as "band". Coupling constants $(J)$ are given in Hertz. Infrared (IR) spectra were obtained using a JASCO FT/IR 460-plus instrument. Optical rotations were determined using a JASCO P1010 polarimeter. MS data were obtained using nano-electrospray ionization in a Micromass Quattro II (triple quad) instrument. Diethyl ether $\left(\mathrm{Et}_{2} \mathrm{O}\right)$, tetrahydrofuran (THF), and dichloromethane $\left(\mathrm{CH}_{2} \mathrm{Cl}_{2}\right)$ were dried by passing through a column of neutral alumina under nitrogen immediately prior to use. Alkylamines, benzene, and toluene were distilled from calcium hydride immediately prior to use. Dimethyl sulfoxide (DMSO) was distilled under reduced pressure from calcium hydride and stored over $4 \AA$ molecular sieves. Anhydrous $N, N$-dimethylformamide (DMF) was purchased from Aldrich chemical company in Sure/Seal ${ }^{\mathrm{TM}}$ bottles. All air and water sensitive reactions were performed under a slight positive pressure of argon. "Evaporated" refers to concentration under reduced pressure. Thin layer chromatography (TLC) was conducted on silica gel $60 \mathrm{~F}_{254}$ TLC plates purchased from EMD Chemicals, Inc. Flash chromatography was carried out using silica gel (60 $\AA, 40$ to $63 \mu \mathrm{m})$ purchased from Sorbent Technologies, Inc.

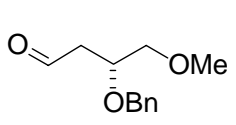

7

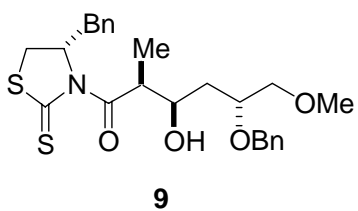

9

Aldol 9. A stirring solution of propionate $8(22.7 \mathrm{~g}$, $85.5 \mathrm{mmol})$ in $\mathrm{CH}_{2} \mathrm{Cl}_{2}(350 \mathrm{~mL})$ was cooled in an ice bath and $\mathrm{TiCl}_{4}(9.40 \mathrm{~mL}, 85.7 \mathrm{mmol})$ was added, dropwise over $5 \mathrm{~min}$, forming a dense yellow-orange precipitate. After stirring for $20 \mathrm{~min}$, (-)-sparteine (19.7 $\mathrm{mL}, 85.7 \mathrm{mmol}$ ) was added, dropwise, causing the suspension to become a homogeneous, deep red-brown colored solution. After stirring for $20 \mathrm{~min}$, 1-methyl-2-pyrrolidinone ( $8.25 \mathrm{~mL}, 86.1 \mathrm{mmol})$ was added, dropwise. After stirring for $20 \mathrm{~min}$ the solution was cooled to $-78{ }^{\circ} \mathrm{C}$ and aldehyde 7 added, as a solution in $\mathrm{CH}_{2} \mathrm{Cl}_{2}(50 \mathrm{~mL}+1 \times 5 \mathrm{~mL}$ rinse $)$. The homogeneous reaction mixture was allowed to stand at $-30{ }^{\circ} \mathrm{C}$ for $14 \mathrm{~h}$. Halfsaturated $\mathrm{NH}_{4} \mathrm{Cl}$ solution was added and the resulting mixture stirred vigorously while warming to rt. The layers were separated and the aqueous solution extracted with $\mathrm{CH}_{2} \mathrm{Cl}_{2}$ (2x). The combined organic solution was dried $\left(\mathrm{Na}_{2} \mathrm{SO}_{4}\right)$ and evaporated. Chromatography, eluting with 28:72 EtOAc/hexanes, afforded $36.0 \mathrm{~g} \mathrm{(90 \% )} \mathrm{of} \mathrm{aldol} 9$ as a bright-yellow colored, viscous oil. ${ }^{1} \mathrm{H} \mathrm{NMR}\left(400 \mathrm{MHz}, \mathrm{CDCl}_{3}\right) \delta 1.29(\mathrm{~d}, J=7.0,3 \mathrm{H})$, 
1.60-1.70 (m, 1H), 1.70-1.83 (m, 1H), $2.88(\mathrm{~d}, J=11.5,1 \mathrm{H}), 3.05(\mathrm{dd}, J=13.0,10.5$, $1 \mathrm{H}), 3.15-3.28$ (band, $2 \mathrm{H}), 3.38(\mathrm{~s}, 3 \mathrm{H}), 3.38(\mathrm{~m}, 1 \mathrm{H}), 3.40-3.60$ (band, $2 \mathrm{H}), 3.85(\mathrm{~m}$, $1 \mathrm{H}), 4.18(\mathrm{~m}, 1 \mathrm{H}), 4.45(\mathrm{~m}, 1 \mathrm{H}), 4.58(\mathrm{~d}, J=11.5,1 \mathrm{H}), 4.72(\mathrm{~d}, J=11.5,1 \mathrm{H}), 5.30(\mathrm{~m}$, 1H), 7.25-7.40 (band, $10 \mathrm{H}) ;{ }^{13} \mathrm{C}$ NMR $\left(100 \mathrm{MHz}, \mathrm{CDCl}_{3}\right) \delta 11.1,32.0,36.59,36.64$, 44.0, 59.1, 68.8, 69.3, 72.2, 74.8, 75.5, 127.2, 127.6, 127.8, 128.3, 128.8, 129.3, 136.4, 138.3, 177.7, 201.1; IR (thin film) $v$ 3436(br), 2927(s), 2877(s), 1694(s), 1495(m), 1455(s), 1343(s), 1260(s), 1165(s), 1136(s), 1028(s); $[\alpha]^{24}=+136\left(\right.$ c $\left.0.65, \mathrm{CH}_{2} \mathrm{Cl}_{2}\right)$; Anal. Calcd for $\mathrm{C}_{25} \mathrm{H}_{31} \mathrm{NO}_{4} \mathrm{~S}_{2}$ : C, 63.39; H, 6.60; N, 2.96. Found: C, 63.22; H, 6.72; N, 3.07 .

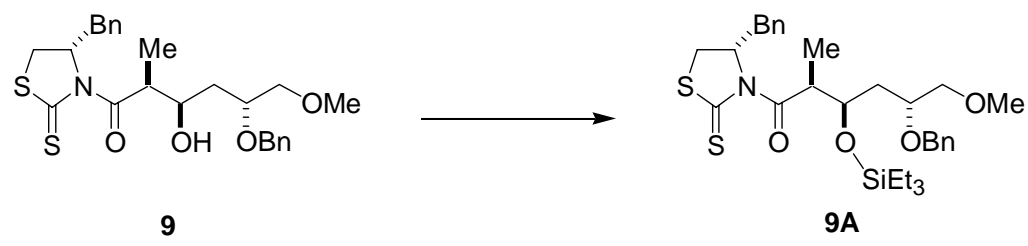

TES ether 9A. A solution of alcohol $9(0.497 \mathrm{~g}, 1.14 \mathrm{mmol})$ and 2,6 -lutidine $(0.199 \mathrm{~mL}$, $1.71 \mathrm{mmol})$ in $\mathrm{CH}_{2} \mathrm{Cl}_{2}(25 \mathrm{~mL})$ was cooled to $0{ }^{\circ} \mathrm{C}$. To the stirring solution was added triethylsilyl trifluoromethanesulfonate $(0.309 \mathrm{~mL}, 1.37 \mathrm{mmol})$. After 5 min saturated $\mathrm{NaHCO}_{3}$ solution was added and the mixture extracted with $\mathrm{CH}_{2} \mathrm{Cl}_{2}(2 \mathrm{x})$. The combined organic solution was dried $\mathrm{Na}_{2} \mathrm{SO}_{4}$ and evaporated. Chromatography, eluting with 12:88 EtOAc/hexanes, afforded $0.608 \mathrm{~g}(97 \%)$ of TES ether 9A as a bright-yellow colored oil. ${ }^{1} \mathrm{H}$ NMR $\left(400 \mathrm{MHz}, \mathrm{CDCl}_{3}\right) \delta 0.57(\mathrm{q}, J=8.0,6 \mathrm{H}), 0.94(\mathrm{t}, J=8.0,9 \mathrm{H}), 1.22(\mathrm{~d}, J=7.0$, $3 \mathrm{H}), 1.72-1.81(\mathrm{~m}, 1 \mathrm{H}), 1.85-1.96(\mathrm{~m}, 1 \mathrm{H}), 2.87(\mathrm{~d}, J=11.5,1 \mathrm{H}), 3.05(\mathrm{dd}, J=13.0$, 11.0, 1H), 3.25-3.32 (band, $2 \mathrm{H}$ ), 3.39 (s, 3H), 3.45-3.53 (band, $2 \mathrm{H}), 3.69$ (m, 1H), 4.20 $(\mathrm{m}, 1 \mathrm{H}), 4.55(\mathrm{~d}, J=12.0,1 \mathrm{H}), 4.64(\mathrm{~m}, 1 \mathrm{H}), 4.77(\mathrm{~d}, J=12.0,1 \mathrm{H}), 5.20(\mathrm{~m}, 1 \mathrm{H}), 7.20-$ $7.40(10 \mathrm{H}) ;{ }^{13} \mathrm{C}$ NMR $\left(100 \mathrm{MHz}, \mathrm{CDCl}_{3}\right) \delta 5.1,6.9,12.6,31.9,36.4,37.6,45.0,59.1$, 69.4, 71.1, 72.1, 75.0, 75.5, 127.1, 127.2, 127.3, 128.8, 129.4, 136.6, 138.9, 176.5, 200.9; IR (thin film) $\vee$ 2956(s), 2877(s), 1700(s), 1497(m), 1455(s), 1341(s), 1260(s); $[\alpha]^{24}=$ +120 (c 0.51, $\mathrm{CH}_{2} \mathrm{Cl}_{2}$ ); Anal. Calcd for $\mathrm{C}_{31} \mathrm{H}_{45} \mathrm{NO}_{4} \mathrm{~S}_{2} \mathrm{Si}$ : C, 63.33; H, 7.71; N, 2.38 . Found: $\mathrm{C}, 63.27 ; \mathrm{H}, 7.64 ; \mathrm{N}, 2.40$.

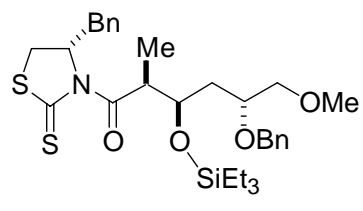

$9 \mathrm{~A}$

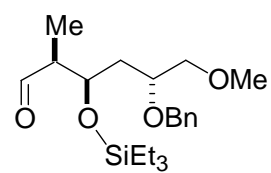

10

Aldehyde 10. A solution of thione 9A $(29.0 \mathrm{~g}, 49.3 \mathrm{mmol})$ in $\mathrm{CH}_{2} \mathrm{Cl}_{2}(400 \mathrm{~mL})$ was cooled to $-78{ }^{\circ} \mathrm{C}$. A solution of $i-\mathrm{Bu}_{2} \mathrm{AlH}$ in heptane $(\sim 1 \mathrm{M})$ was added dropwise until the yellow color disappeared $(95 \mathrm{~mL}, \sim 95 \mathrm{mmol})$. A saturated solution of sodium potassium tartrate $(200 \mathrm{~mL})$ was added and the mixture allowed to warm to $\mathrm{rt}$. Water 
$(100 \mathrm{~mL})$ and $\mathrm{CH}_{2} \mathrm{Cl}_{2}(100 \mathrm{~mL})$ were added and the mixture stirred vigorously for $1 \mathrm{~h}$. The layers were separated and the aqueous solution extracted with $\mathrm{CH}_{2} \mathrm{Cl}_{2}(2 \times 100 \mathrm{~mL})$. The combined organic solution was dried $\left(\mathrm{Na}_{2} \mathrm{SO}_{4}\right)$ and evaporated. The residue was mixed with hexanes, whereupon the (S)-4-(phenylmethyl)-2-thiazolidinethione precipitated as a white solid (after seeding). The solid was filtered off and washed with additional hexanes. The combined hexanes solution was evaporated. Chromatography, eluting with 12:88 EtOAc/hexanes, afforded $16.2 \mathrm{~g} \mathrm{(86 \% )}$ of aldehyde $\mathbf{1 0}$ as a colorless oil. ${ }^{1} \mathrm{H}$ NMR $\left(400 \mathrm{MHz}, \mathrm{CDCl}_{3}\right) \delta 0.57$ (q, $\left.J=8.0,6 \mathrm{H}\right), 0.93(\mathrm{t}, J=8.0,9 \mathrm{H}), 1.04(\mathrm{~d}, J=$ 7.0, 3H), 1.62 (ddd, $J=14.5,8.5,3.5,1 \mathrm{H}), 1.80$ (ddd, $J=14.0,9.0,4.5,1 \mathrm{H}), 2.48$ (m, $1 \mathrm{H}$ ), 3.36 (s, 3H), 3.43-3.52 (band, $2 \mathrm{H}$ ), 3.68 (dddd, $J=9.0,3 \times 4.5,1 \mathrm{H}$ ), 4.32 (dddd, $\mathrm{J}=$ $4 \times 4.0,1 \mathrm{H}), 4.52(\mathrm{~d}, J=11.5,1 \mathrm{H}), 4.75(\mathrm{~d}, J=11.5,1 \mathrm{H}), 7.25-7.35$ (band, $5 \mathrm{H}), 9.79(\mathrm{~s}$, $1 \mathrm{H}) ;{ }^{13} \mathrm{C}$ NMR $\left(100 \mathrm{MHz}, \mathrm{CDCl}_{3}\right) \delta 5.1,6.8,7.9,37.4,52.3,59.1,69.7,71.5,75.1,75.2$, 127.5, 128.3, 138.6, 205.1; IR (thin film) $\vee$ 2954(s), 2876(s), 1726(s), 1456(s), 1239(m), 1107(s), 1030(s), 739(s); $[\alpha]_{\mathrm{D}}^{25}=+84\left(\mathrm{c} 4.6, \mathrm{CH}_{2} \mathrm{Cl}_{2}\right)$; MS (ESI) calculated for $\mathrm{C}_{21} \mathrm{H}_{36} \mathrm{NaO}_{4} \mathrm{Si}[\mathrm{M}+\mathrm{Na}]^{+}: \mathrm{m} / \mathrm{z}$ 403.5, found: $\mathrm{m} / \mathrm{z} 403.2$.

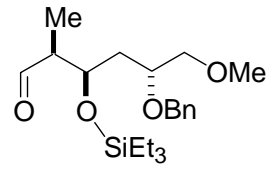

10

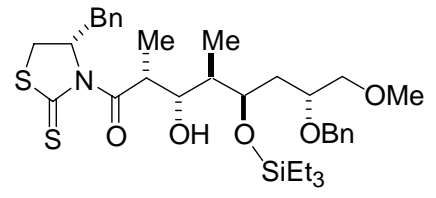

11

Aldol 11. A stirring solution of propionate $8(12.0 \mathrm{~g}, 45.2 \mathrm{mmol})$ in $\mathrm{CH}_{2} \mathrm{Cl}_{2}(200 \mathrm{~mL})$ was cooled in an ice bath and $\mathrm{TiCl}_{4}(5.00 \mathrm{~mL}, 45.6 \mathrm{mmol})$ was added, dropwise, over 5 $\mathrm{min}$, forming a dense yellow-orange precipitate. After stirring for $20 \mathrm{~min}, i-\mathrm{Pr}_{2} \mathrm{NEt}(19.0$ $\mathrm{mL}, 109.1 \mathrm{mmol}$ ) was added, dropwise, causing the suspension to become a homogeneous, deep red-brown colored solution. After stirring for 20 min the solution was cooled to $-78^{\circ} \mathrm{C}$ and aldehyde 10 added over 15 min, as a solution in $\mathrm{CH}_{2} \mathrm{Cl}_{2}(50 \mathrm{~mL}$ $+1 \times 5 \mathrm{~mL}$ rinse). The homogeneous reaction mixture was allowed to stand at $-30{ }^{\circ} \mathrm{C}$ for $13 \mathrm{~h}$. The reaction mixture was poured into a vigorously stirring $\mathrm{NaHCO}_{3}$ solution. The mixture was filtered through celite, and the layers separated. The aqueous solution was extracted with $\mathrm{CH}_{2} \mathrm{Cl}_{2}(3 \mathrm{x})$ and the combined organic solution dried $\left(\mathrm{Na}_{2} \mathrm{SO}_{4}\right)$ and evaporated. Chromatography, eluting with 18:82 EtOAc/hexanes, afforded $17.1 \mathrm{~g} \mathrm{(62 \% )}$ of aldol 11 as a bright-yellow colored, viscous oil. ${ }^{1} \mathrm{H}$ NMR $\left(400 \mathrm{MHz}, \mathrm{CDCl}_{3}\right) \delta 0.61$ (qm, $J=8.0,6 \mathrm{H}), 0.83$ (d, $J=7.0,3 \mathrm{H}), 0.95(\mathrm{t}, J=8.0,9 \mathrm{H}), 1.19$ (d, $J=7.0,3 \mathrm{H}), 1.68$ 1.89 (band, $3 \mathrm{H}$ ), 2.88 (dd, $J=11.5,1.0,1 \mathrm{H}), 3.02$ (dd, $J=13.0,11.0,1 \mathrm{H}), 3.26-3.38$ (band, 2H), 3.38 (s, 3H), 3.46-3.56 (band, 2H), 3.69 (m, 1H), 4.09 (br. s, 1H), 4.17 (m, $1 \mathrm{H}), 4.25(\mathrm{dm}, J=10.0,1 \mathrm{H}), 4.54(\mathrm{~d}, J=12.0,1 \mathrm{H}), 4.75(\mathrm{~m}, 1 \mathrm{H}), 4.78(\mathrm{~d}, J=12.0,1 \mathrm{H})$, $5.42(\mathrm{~m}, 1 \mathrm{H}), 7.23-7.38$ (band, $10 \mathrm{H}) ;{ }^{13} \mathrm{C} \mathrm{NMR}\left(100 \mathrm{MHz}, \mathrm{CDCl}_{3}\right)$ 8 5.0, 6.9, 9.6, 12.2, $31.7,35.4,37.0,40.4,41.8,59.1,69.3,71.2,71.9,73.2,75.3,75.6,127.1,127.3,128.2$, 128.8, 129.4, 136.6, 138.9, 177.4, 200.9; IR (thin film) v 3456(br), 2954(s), 2875(s), 1704(s), 1678(s), 1455(s), 1343(s), 1260(s), 1163(s), 1135(s), 743(s); [ $\alpha]^{24}{ }_{\mathrm{D}}=+115(\mathrm{c}$ 3.2, $\mathrm{CH}_{2} \mathrm{Cl}_{2}$ ); Anal. Calcd for $\mathrm{C}_{34} \mathrm{H}_{51} \mathrm{NO}_{5} \mathrm{~S} \mathrm{~S}_{2} \mathrm{Si}$ : C, 63.22; H, 7.96; N, 2.17. Found: $\mathrm{C}$, 63.19; H, 8.03; N, 2.31 . 


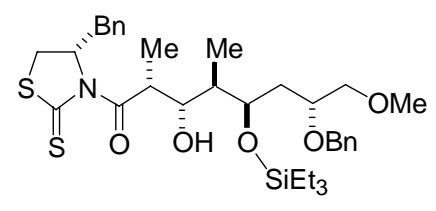

11

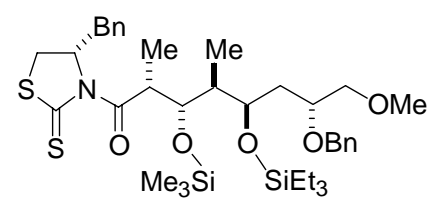

$11 \mathrm{~A}$

TMS ether 11A. A solution of aldol $11(17.1 \mathrm{~g}, 26.5 \mathrm{mmol})$ in $\mathrm{CH}_{2} \mathrm{Cl}_{2}(50 \mathrm{~mL})$ was cooled to $0{ }^{\circ} \mathrm{C}$. To the stirring solution was added $\mathrm{Et}_{3} \mathrm{~N}(4.80 \mathrm{~mL}, 34.4 \mathrm{mmol})$, trimethylsilyl chloride $(4.05 \mathrm{~mL}, 31.9 \mathrm{mmol})$, and then DMAP $(0.175 \mathrm{~g}, 1.43 \mathrm{mmol})$. After $1.5 \mathrm{~h}$ saturated $\mathrm{NaHCO}_{3}$ solution was added and the mixture was stirred vigorously. Water and $\mathrm{CH}_{2} \mathrm{Cl}_{2}$ were added to the mixture then the layers were separated and the aqueous solution extracted with $\mathrm{CH}_{2} \mathrm{Cl}_{2}(2 \times 30 \mathrm{~mL})$. The combined organic solution was dried $\left(\mathrm{Na}_{2} \mathrm{SO}_{4}\right)$ and evaporated. Chromatography, eluting with 9:91 EtOAc/hexanes, afforded $15.0 \mathrm{~g}(79 \%)$ of TMS ether 11A as a bright-yellow colored, viscous oil. ${ }^{1} \mathrm{H}$ $\operatorname{NMR}\left(500 \mathrm{MHz}, \mathrm{CDCl}_{3}\right) \delta 0.17(\mathrm{~s}, 9 \mathrm{H}), 0.63(\mathrm{q}, J=8.0,6 \mathrm{H}), 0.83(\mathrm{~d}, J=7.0,3 \mathrm{H}), 0.98$ $(\mathrm{t}, J=8.0,9 \mathrm{H}), 1.12(\mathrm{~d}, J=7.0,3 \mathrm{H}), 1.57(\mathrm{~m}, 1 \mathrm{H}), 1.78(\mathrm{ddd}, J=14.0,5.5,5.5,1 \mathrm{H})$, 1.87 (ddd, $J=14.0,8.5,6.5,1 \mathrm{H}), 2.85(\mathrm{~d}, J=11.5,1 \mathrm{H}), 3.03(\mathrm{dd}, J=13.0,11.0,1 \mathrm{H})$, 3.28 (dd, $J=13.5,3.5,1 \mathrm{H}), 3.33$ (dd, $J=12.5,7.0,1 \mathrm{H}), 3.38$ (s, 3H), 3.42-3.54 (band, $3 \mathrm{H}), 4.12(\mathrm{ddd}, J=8.0,5.0,1.5,1 \mathrm{H}), 4.37(\mathrm{dd}, J=8.0,3.0,1 \mathrm{H}), 4.56(\mathrm{~d}, J=12.0,1 \mathrm{H})$, 4.62 (dddd, $J=3 \times 7.0,3.5,1 \mathrm{H}), 4.67$ (d, $J=12.0,1 \mathrm{H}), 5.23(\mathrm{ddd}, J=10.5,7.0,3.5,1 \mathrm{H})$, 7.23-7.38 (band, $10 \mathrm{H}$ ); ${ }^{13} \mathrm{C}$ NMR $\left(100 \mathrm{MHz}, \mathrm{CDCl}_{3}\right) \delta 1.3,6.0,7.2,10.1,12.2,31.8$, $36.8,37.6,42.0,43.0,59.1,69.6,69.9,71.6,73.4,74.9,75.9,127.1,127.4,127.7,128.2$, 128.9, 129.4, 136.8, 138.7, 177.0, 200.7; IR (thin film) $\vee 2954(\mathrm{~s}), 2875(\mathrm{~s}), 1698(\mathrm{~s})$,

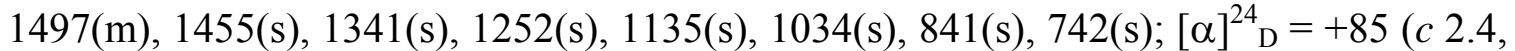
$\mathrm{CH}_{2} \mathrm{Cl}_{2}$ ); MS (ESI) calculated for $\mathrm{C}_{37} \mathrm{H}_{59} \mathrm{NNaO}_{5} \mathrm{~S}_{2} \mathrm{Si}_{2} \quad[\mathrm{M}+\mathrm{Na}]^{+}: \mathrm{m} / \mathrm{z} 740.3$, found: $\mathrm{m} / \mathrm{z}$ 740.6 .
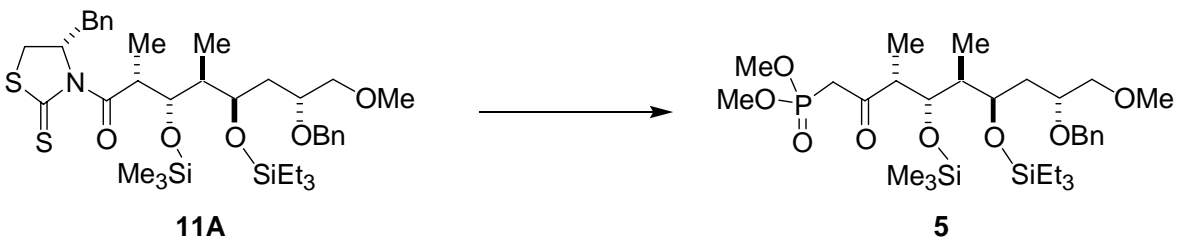

Phosphonate 5. A stirring solution of dimethyl methylphosphonate $(0.665 \mathrm{~mL}, 6.22$ $\mathrm{mmol})$ in THF $(130 \mathrm{~mL})$ was cooled to $-78{ }^{\circ} \mathrm{C}$. A solution of BuLi (33 mL (2.5 $\mathrm{M} /$ hexanes), $82.5 \mathrm{mmol}$ ) was added, dropwise, over $10 \mathrm{~min}$ and the resulting solution stirred for $1 \mathrm{~h}$. A solution of TMS ether 11A $(15.0 \mathrm{~g}, 20.9 \mathrm{mmol})$ in THF $(35 \mathrm{~mL}+2 \times 17$ $\mathrm{mL}$ rinses) was added, dropwise, to the reaction mixture. After $1 \mathrm{~h}$ no yellow color was visible. After $2 \mathrm{~h}$ a solution of saturated $\mathrm{NH}_{4} \mathrm{Cl}$ was added and the stirring mixture allowed to warm to rt. The mixture was extracted with $\mathrm{Et}_{2} \mathrm{O}(3 \times 200 \mathrm{~mL})$ and the combined organic solution dried $\left(\mathrm{Na}_{2} \mathrm{SO}_{4}\right)$ and evaporated. Chromatography, eluting with 55:45 EtOAc/hexanes, afforded $12.7 \mathrm{~g}(96 \%)$ of phosphonate 5 as a colorless, viscous oil. ${ }^{1} \mathrm{H}$ NMR $\left(500 \mathrm{MHz}, \mathrm{CDCl}_{3}\right) \delta 0.05(\mathrm{~s}, 9 \mathrm{H}), 0.59(\mathrm{dq}, J=7.5,2.0,6 \mathrm{H}), 0.76$ $(\mathrm{d}, J=7.0,3 \mathrm{H}), 0.95(\mathrm{t}, J=8.0,9 \mathrm{H}), 0.98(\mathrm{~d}, J=6.5,3 \mathrm{H}), 1.52(\mathrm{~m}, 1 \mathrm{H}), 1.75-1.87$ (band, 
2H), 2.88 (qm, $J=9.0,1 \mathrm{H}), 2.97$ (dd, $J=23.0,14.0,1 \mathrm{H}), 3.29$ (dd, $J=23.0,14.0,1 \mathrm{H}$ ), 3.35 (s, 3H), 3.38-3.50 (band, 3H), 3.74 (s, 3H), 3.77 (s, 3H), 4.05-4.10 (band, $2 \mathrm{H}$ ), 4.51 $(\mathrm{d}, J=12.5,1 \mathrm{H}), 4.66(\mathrm{~d}, J=12.5,1 \mathrm{H}), 7.22-7.35$ (band, $5 \mathrm{H}) ;{ }^{13} \mathrm{C}$ NMR $(125 \mathrm{MHz}$, $\left.\mathrm{CDCl}_{3}\right) \delta 0.80,6.0,7.1,8.6,9.6,37.6,39.5(\mathrm{~d}, J=128), 42.4,50.5,52.8(\mathrm{~d}, J=6.0), 53.0$ (d, $J=6.5), 59.1,69.9,71.6,73.5,74.7,75.8,127.4,127.6,128.2,138.5,203.9$ (d, $J=$ 6.5); IR (thin film) v 2954(s), 1714(s), 1456(s), 1252(s), 1032(s), 840(s), 741(s); $[\alpha]^{27}{ }_{\mathrm{D}}=$ -45 (c 3.9, $\mathrm{CH}_{2} \mathrm{Cl}_{2}$ ); MS (ESI) calculated for $\mathrm{C}_{30} \mathrm{H}_{58} \mathrm{O}_{8} \mathrm{PSi}_{2}[\mathrm{M}+\mathrm{H}]^{+}: \mathrm{m} / \mathrm{z}$ 633.3, found: $\mathrm{m} / \mathrm{z} 633.4$.
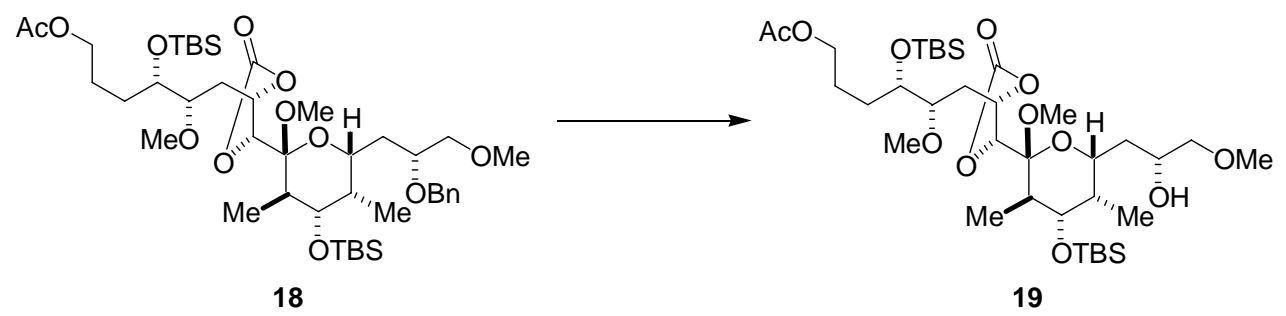

Alcohol 19. To a solution of benzyl ether $18(0.500 \mathrm{mg}, 0.606 \mathrm{mmol})$ in EtOAc was added $\mathrm{Pd} / \mathrm{C}(10$ wt \%) $(0.500 \mathrm{~g})$. The mixture was stirred vigorously under an atmosphere of $\mathrm{H}_{2}$ (ambient pressure). After $5 \mathrm{~h} \mathrm{TLC}$ analysis indicated that reaction was complete. The mixture was filtered through a pad of celite, washing with EtOAc, and the solution evaporated. Chromatography, eluting with 3:5 EtOAc/hexanes, afforded $0.455 \mathrm{~g}$ $(100 \%)$ of alcohol 19 as a colorless oil. ${ }^{1} \mathrm{H}$ NMR $\left(500 \mathrm{MHz}, \mathrm{CDCl}_{3}\right) \delta 0.01(\mathrm{~s}, 3 \mathrm{H}), 0.04$ (s, 3H), 0.06 (s, 3H), 0.09 (s, 3H), 0.84 (d, $J=7.0,3 \mathrm{H}), 0.88$ (br. s, 18H), 0.97 (d, $J=6.5$, $3 \mathrm{H}), 1.27(\mathrm{~m}, 1 \mathrm{H}), 1.33(\mathrm{~m}, 1 \mathrm{H}), 1.53-1.67$ (band, $4 \mathrm{H}), 1.70(\mathrm{~m}, 1 \mathrm{H}), 1.77(\mathrm{~m}, 1 \mathrm{H}), 1.91$ 1.99 (band, $2 \mathrm{H}$ ), 2.04 (s, 3H), 2.37 (br. d, $J=3.0,1 \mathrm{H}), 3.20$ (dd, $J=9.5,8.5,1 \mathrm{H}), 3.27$ (s, $3 \mathrm{H}), 3.37-3.43$ (band, $2 \mathrm{H}), 3.38$ (s, $3 \mathrm{H}), 3.39$ (s, 3H), $3.76(\mathrm{dd}, J=10.5,4.5,1 \mathrm{H}), 3.84$ (m, 1H), 3.95 (m, 1H), 4.02-4.09 (band, 3H), 4.33 (d, $J=5.0,1 \mathrm{H}), 4.95$ (dm, $J=10.5$, $1 \mathrm{H}) ;{ }^{13} \mathrm{C}$ NMR $\left(125 \mathrm{MHz}, \mathrm{CDCl}_{3}\right) \delta-4.9,-4.7,-4.4,-4.3,5.0,11.5,17.9,18.0,21.0$, 25.4, 25.7, 25.8, 27.2, 34.5, 35.4, 36.1, 39.3, 48.8, 58.5, 59.0, 64.4, 66.7, 67.6, 70.3, 73.2, 75.2, 77.3, 79.4, 81.1, 100.0, 153.9, 171.1; IR (thin film) v 3511(br), 2954(s), 2857(s), 1814(s), 1740(s), 1463(m), 1365(m), 1252(s), 1073(s), 837(s), 775(s); $[\alpha]^{26}{ }_{\mathrm{D}}=+13(\mathrm{c}$ 3.4, $\mathrm{CH}_{2} \mathrm{Cl}_{2}$ ); MS (ESI) calculated for $\mathrm{C}_{36} \mathrm{H}_{70} \mathrm{NaO}_{12} \mathrm{Si}_{2}[\mathrm{M}+\mathrm{Na}]^{+}: \mathrm{m} / \mathrm{z} 773.4$, found: $\mathrm{m} / \mathrm{z}$ 773.5 .

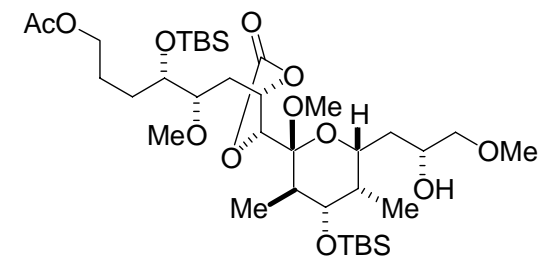

19

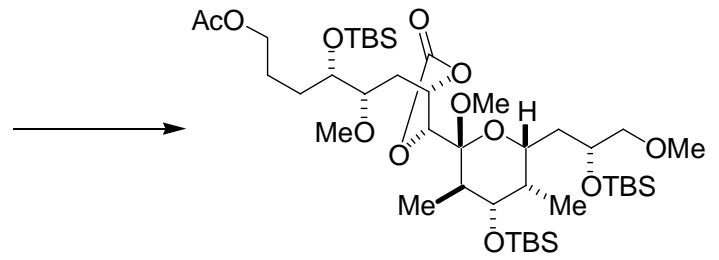

20

TBS ether 20. A solution of alcohol $19(0.316 \mathrm{~g}, 0.421 \mathrm{mmol})$ and 2,6-lutidine $(0.100$ $\mathrm{mL}, 0.859 \mathrm{mmol})$, in $\mathrm{CH}_{2} \mathrm{Cl}_{2}(5 \mathrm{~mL})$, was cooled to $-78^{\circ} \mathrm{C}$. To the stirring solution was added TBSOTf $(0.150 \mathrm{~mL}, 0.653 \mathrm{mmol})$ dropwise over $2 \mathrm{~min}$. After $1.5 \mathrm{~h}$ TLC analysis 
indicated that the reaction was complete. Saturated $\mathrm{NaHCO}_{3}$ solution was added $(5 \mathrm{~mL})$ and the mixture was allowed to warm to $\mathrm{rt}$ and the phases separated. The aqueous solution was extracted with $\mathrm{CH}_{2} \mathrm{Cl}_{2}(3 \mathrm{x})$. The combined organic solution was dried $\left(\mathrm{Na}_{2} \mathrm{SO}_{4}\right)$ and evaporated. Chromatography, eluting with EtOAc/hexanes, afforded 0.351 $\mathrm{g}(96 \%)$ of TBS ether 20 as a colorless oil. ${ }^{1} \mathrm{H}$ NMR $\left(400 \mathrm{MHz}, \mathrm{CDCl}_{3}\right) \delta 0.02(\mathrm{~s}, 3 \mathrm{H})$, 0.05 (s, 3H), 0.05 (band, 6H), 0.06 (s, 3H), 0.09 (s, 3H), 0.84 (d, $J=6.8,3 \mathrm{H}), 0.87$ (s, $9 \mathrm{H}), 0.87(\mathrm{~s}, 9 \mathrm{H}), 0.88(\mathrm{~s}, 9 \mathrm{H}), 0.98(\mathrm{~d}, J=6.4,3 \mathrm{H}) ; 1.27$ (m, $1 \mathrm{H}), 1.47$ (ddd, $J=14.4$, 8.0, 3.2, 1H), 1.53-1.84 (band, $6 \mathrm{H}$ ), 1.88-2.02 (band, $2 \mathrm{H}$ ), 2.04 (s, 3H), 3.21-3.33 (band, $2 \mathrm{H}), 3.24(\mathrm{~s}, 3 \mathrm{H}), 3.30(\mathrm{~s}, 3 \mathrm{H}), 3.38(\mathrm{~m}, 1 \mathrm{H}), 3.39(\mathrm{~s}, 3 \mathrm{H}), 3.70(\mathrm{dd}, J=10.4,4.4,1 \mathrm{H})$, 3.82-3.92 (band, 3H), 4.03-4.09 (band $2 \mathrm{H}), 4.36(\mathrm{~d}, J=4.8,1 \mathrm{H}), 4.88(\mathrm{~m}, 1 \mathrm{H}) ;{ }^{13} \mathrm{C}$ NMR $\left(100 \mathrm{MHz}, \mathrm{CDCl}_{3}\right) \delta-4.8,-4.7,-4.7,-4.4,-4.4,-3.8,4.8,11.6,17.9,18.1,18.2$, $20.9,25.4,25.7,25.8,25.9,27.1,34.5,35.1,38.5,39.6,48.8,58.3,58.7,64.5,69.1,69.7$, 70.2, 73.4, 75.6, 77.6, 79.4, 80.6, 100.1, 153.9, 171.1; IR (thin film) $\vee 2954(\mathrm{~s}), 2929(\mathrm{~s})$, 2890(s), 2857(s), 1817(s), 1741(s), 1462(m), 1363(m), 1251(s), 1072(s), 836(s), 775(s); $[\alpha]^{26}=+8.9\left(\right.$ c 3.9, $\mathrm{CH}_{2} \mathrm{Cl}_{2}$ ); MS (ESI) calculated for $\mathrm{C}_{42} \mathrm{H}_{84} \mathrm{NaO}_{12} \mathrm{Si}_{3} \quad[\mathrm{M}+\mathrm{Na}]^{+}: \mathrm{m} / \mathrm{z}$ 887.5, found: $\mathrm{m} / \mathrm{z} 887.6$.
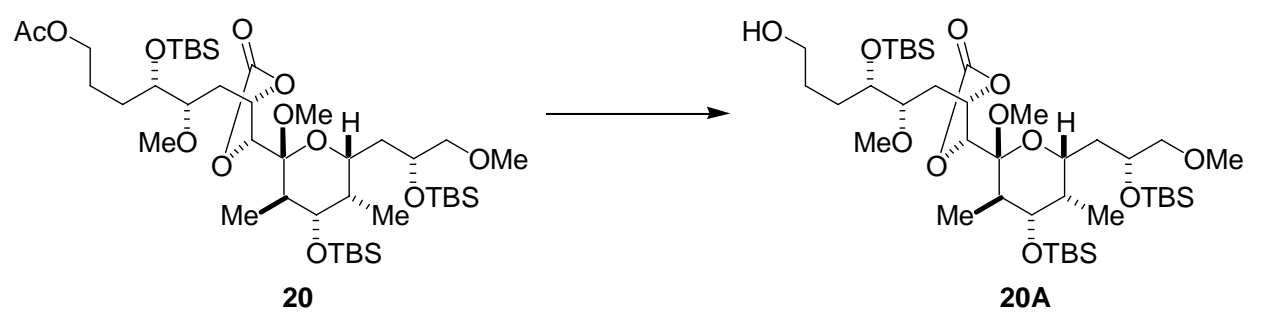

Alcohol 20A. A solution of acetate $20(351 \mathrm{mg}, 0.406 \mathrm{mmol})$ in $\mathrm{MeOH}(4 \mathrm{~mL})$ was cooled in an ice-water bath. Powdered $\mathrm{K}_{2} \mathrm{CO}_{3}(0.2 \mathrm{~g})$ was added in one portion and the resulting heterogeneous mixture stirred vigorously. The cooling bath temperature was maintained between $10-15^{\circ} \mathrm{C}$. After $5 \mathrm{~h} \mathrm{TLC}$ analysis indicated that the desired reaction was complete. Saturated $\mathrm{NaHCO}_{3}$ solution $(10 \mathrm{~mL})$, water $(10 \mathrm{~mL})$ and $\mathrm{CH}_{2} \mathrm{Cl}_{2}(10 \mathrm{~mL})$ were added and the mixture was shaken and separated. The aqueous solution was extracted with $\mathrm{CH}_{2} \mathrm{Cl}_{2}(3 \mathrm{x})$ and the combined organic solution was dried $\left(\mathrm{Na}_{2} \mathrm{SO}_{4}\right)$ and evaporated. Chromatography with 28:72 EtOAc/hexanes afforded $312 \mathrm{mg}(93 \%)$ of alcohol 20A as a colorless oil. ${ }^{1} \mathrm{H}$ NMR $\left(500 \mathrm{MHz}, \mathrm{CDCl}_{3}\right) \delta 0.02(\mathrm{~s}, 3 \mathrm{H}), 0.05(\mathrm{~s}, 3 \mathrm{H})$, 0.06 (band, 6H), 0.07 (s, 3H), 0.09 (s, 3H), 0.84 (d, $J=7.0,3 \mathrm{H}), 0.87$ (s, 9H), 0.878 (s, 9H), $0.883(\mathrm{~s}, 9 \mathrm{H}), 0.98(\mathrm{~d}, J=6.5,3 \mathrm{H}), 1.30(\mathrm{~m}, 1 \mathrm{H}), 1.44-1.77$ (band, $8 \mathrm{H}), 1.92(\mathrm{~m}$, $1 \mathrm{H}), 1.96(\mathrm{~m}, 1 \mathrm{H}), 3.23-3.32$ (band, $2 \mathrm{H}), 3.24(\mathrm{~s}, 3 \mathrm{H}), 3.31(\mathrm{~s}, 3 \mathrm{H}), 3.38(\mathrm{~m}, 1 \mathrm{H}), 3.39(\mathrm{~s}$, $3 \mathrm{H}$ ), 3.64 (band, $2 \mathrm{H}$ ), 3.70 (dd, $J=10.5,4.5,1 \mathrm{H}$ ), 3.84-3.91 (band, $3 \mathrm{H}$ ), 4.35 (d, $J=5.0$, $1 \mathrm{H}), 4.88$ (ddd, $J=10.5,5.0,3.0,1 \mathrm{H}) ;{ }^{13} \mathrm{C}$ NMR $\left(125 \mathrm{MHz}, \mathrm{CDCl}_{3}\right) \delta-4.8,-4.7,-4.6$, $4.4,-4.3,-3.8,4.8,11.6,18.0,18.1,18.2,25.7,25.8,25.9,27.1,29.5,34.6,35.1,38.5$, 39.6, 48.7, 58.3, 58.8, 63.0, 69.0, 69.7, 70.6, 73.4, 75.6, 77.6, 79.4, 80.6, 100.1, 153.9; IR (thin film) $v$ 3465(br), 2929(s), 2857(s), 1816(s), 1463(m), 1386(m), 1255(s), 1071(s), 836(s), 775(s); $[\alpha]_{\mathrm{D}}^{25}=+11\left(\mathrm{c} 4.2, \mathrm{CH}_{2} \mathrm{Cl}_{2}\right)$; MS (ESI) calculated for $\mathrm{C}_{40} \mathrm{H}_{82} \mathrm{NaO}_{11} \mathrm{Si}_{3}[\mathrm{M}+\mathrm{Na}]^{+}: \mathrm{m} / \mathrm{z}$ 845.5, found: $\mathrm{m} / \mathrm{z}$ 845.6. 


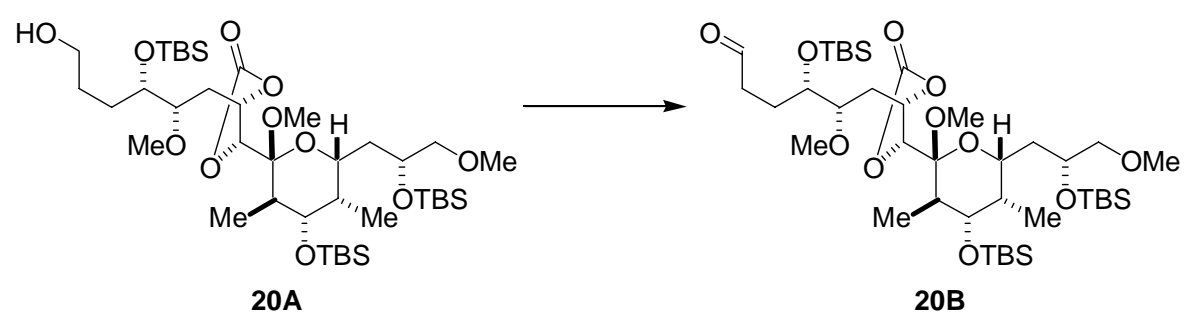

Aldehyde 20B. A stirring solution of dimethyl sulfoxide $(0.060 \mathrm{~mL}, 0.85 \mathrm{mmol})$ in $\mathrm{CH}_{2} \mathrm{Cl}_{2}(2.0 \mathrm{~mL})$ was cooled in a dry ice/acetone bath. Oxalyl chloride $(0.35 \mathrm{~mL}$ of a 2.0 $\mathrm{M}$ solution in $\mathrm{CH}_{2} \mathrm{Cl}_{2}, 0.70 \mathrm{mmol}$ ) was added over $5 \mathrm{~min}$. After stirring at $-78^{\circ} \mathrm{C}$ for 20 min alcohol 20A $(312 \mathrm{mg}, 0.379 \mathrm{mmol})$ was added as a solution in $\mathrm{CH}_{2} \mathrm{Cl}_{2}(1 \mathrm{~mL}+2 \mathrm{x}$ $0.5 \mathrm{~mL}$ rinses $)$ over $5 \mathrm{~min}$. After stirring at $-78^{\circ} \mathrm{C}$ for $20 \mathrm{~min} \mathrm{Et}_{3} \mathrm{~N}$ was added $(0.33 \mathrm{~mL}$, $2.4 \mathrm{mmol}$ ) over $2 \mathrm{~min}$ and the reaction mixture was allowed to stir for $15 \mathrm{~min}$ at $-78{ }^{\circ} \mathrm{C}$ before being allowed to warm to rt. The reaction mixture was shaken with $0.2 \mathrm{~N}$ $\mathrm{NaHSO}_{4}(2 \mathrm{~mL})$ and the layers separated. The aqueous solution was extracted with $\mathrm{CH}_{2} \mathrm{Cl}_{2}$ (3x) and the combined organic solution dried $\left(\mathrm{Na}_{2} \mathrm{SO}_{4}\right)$ and evaporated. Chromatography, eluting with 13:87 EtOAc/hexanes, afforded 0.291 g (94\%) of aldehyde 20B as a colorless oil. ${ }^{1} \mathrm{H}$ NMR $\left(400 \mathrm{MHz}, \mathrm{CDCl}_{3}\right) \delta 0.02(\mathrm{~s}, 3 \mathrm{H}), 0.05(\mathrm{~s}, 3 \mathrm{H}), 0.06$ (band, 9H), 0.09 (s, 3H), 0.84 (d, J=7.0, 3H), 0.87 (s, 9H), 0.873 (s, 9H), 0.88 (s, 9H), 0.98 (d, $J=6.5,1 \mathrm{H}), 1.43-1.57$ (band, $2 \mathrm{H}$ ), 1.61-1.72 (band, $3 \mathrm{H}$ ), 1.86-2.02 (band, $3 \mathrm{H}$ ), 2.42-2.62 (band, $2 \mathrm{H}$ ), 3.22-3.32 (band, $2 \mathrm{H}), 3.25$ (s, 3H), $3.30(\mathrm{~s}, 3 \mathrm{H}), 3.39(\mathrm{~m}, 1 \mathrm{H}), 3.40$ (s, 3H), $3.71(\mathrm{dd}, J=10.0,4.5,1 \mathrm{H}), 3.85-3.93$ (band, $3 \mathrm{H}), 4.36(\mathrm{~d}, J=5.0,1 \mathrm{H}), 4.88$ (ddd, $J=10.5,5.0,2.5,1 \mathrm{H}), 9.78(\mathrm{~s}, 1 \mathrm{H}) ;{ }^{13} \mathrm{C}$ NMR $\left(100 \mathrm{MHz}, \mathrm{CDCl}_{3}\right) \delta-4.9,-4.7,-4.4$, -3.8, 4.8, 11.6, 17.9, 18.1, 18.2, 22.9, 25.7, 25.8, 25.9, 34.4, 35.1, 38.5, 39.6, 40.5, 48.7, $58.2,58.7,69.0,69.3,69.7,73.4,75.5,77.6,79.2,80.6,100.0,153.8,201.9$; IR (thin film) $v$ 2930(s), 2889(s), 2857(s), 2712(w), 1816(s), 1727(m), 1463(m), 1388(m), 1254(s), 1073(s), 837(s), 775(s); $[\alpha]^{27}=+9.8\left(c 3.9, \mathrm{CH}_{2} \mathrm{Cl}_{2}\right)$; MS (ESI) calculated for $\mathrm{C}_{40} \mathrm{H}_{80} \mathrm{NaO}_{11} \mathrm{Si}_{3}[\mathrm{M}+\mathrm{Na}]^{+}: \mathrm{m} / \mathrm{z}$ 843.5, found: $\mathrm{m} / \mathrm{z}$ 843.6.

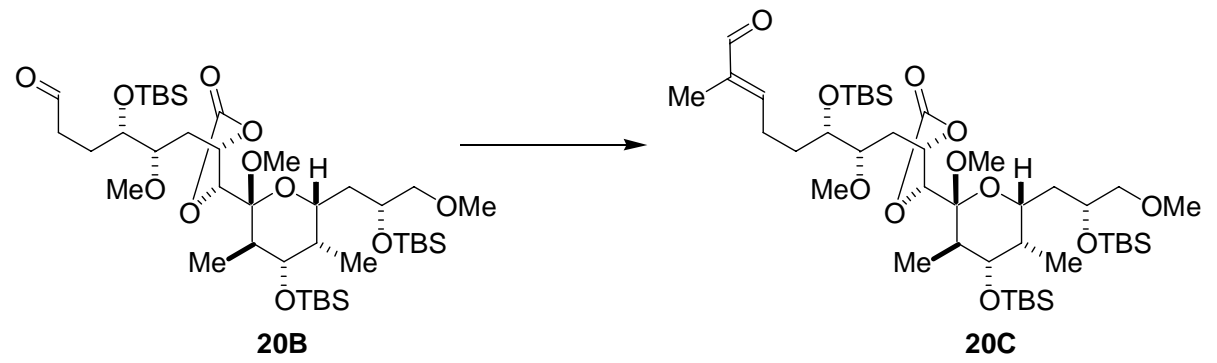

Aldehyde 20C. A stirring mixture of aldehyde 20B $(0.291 \mathrm{~g}, 0.354 \mathrm{mmol})$ and $(\alpha-$ formylethylidene)triphenylphosphorane (25) $(0.225 \mathrm{~g}, 0.707 \mathrm{mmol})$ in chlorobenzene $\left(3.0 \mathrm{~mL}\right.$ ) was heated at $90{ }^{\circ} \mathrm{C}$ (the solution became homogeneous at this temperature). More phosphorane $(0.100 \mathrm{~g}, 0.314 \mathrm{mmol})$ was added after $13.5 \mathrm{~h}$. After another $3 \mathrm{~h}{ }^{1} \mathrm{H}$ NMR analysis indicated that the reaction was complete. The solution was allowed to cool then evaporated to dryness. The orange/brown solid was boiled in hexanes and after 
cooling the solid was filtered off and washed with hexanes. The combined hexanes solution was evaporated and chromatography, eluting with 14:86 EtOAc/hexanes, afforded $0.238 \mathrm{~g}(78 \%)$ of aldehyde $20 \mathrm{C}$ as a colorless oil. ${ }^{1} \mathrm{H}$ NMR $\left(400 \mathrm{MHz}, \mathrm{CDCl}_{3}\right)$ $\delta 0.02(\mathrm{~s}, 3 \mathrm{H}), 0.05$ (band, $9 \mathrm{H}), 0.08(\mathrm{~s}, 3 \mathrm{H}), 0.11(\mathrm{~s}, 3 \mathrm{H}), 0.84(\mathrm{~d}, J=7.0,3 \mathrm{H}), 0.87(\mathrm{~s}$, 9H), 0.88 (s, 9H), 0.89 (s, 9H), 0.98 (d, $J=7.0,3 \mathrm{H}), 1.36-1.52$ (band, $2 \mathrm{H}$ ), 1.59-1.77 (band, $4 \mathrm{H}), 1.74(\mathrm{~s}, 3 \mathrm{H}), 1.87-2.03$ (band, $2 \mathrm{H}), 2.29(\mathrm{~m}, 1 \mathrm{H}), 2.51(\mathrm{~m}, 1 \mathrm{H}), 3.21-3.31$ (band, $2 \mathrm{H}), 3.24(\mathrm{~s}, 3 \mathrm{H}), 3.29(\mathrm{~s}, 3 \mathrm{H}), 3.40(\mathrm{~m}, 1 \mathrm{H}), 3.41(\mathrm{~s}, 3 \mathrm{H}), 3.70(\mathrm{dd}, J=10.5,4.5$, 1H), 3.85-3.94 (band, 3H), 4.35 (d, $J=5.0,1 \mathrm{H}), 4.88$ (ddd, $J=11.0,4.0,2.5,1 \mathrm{H}$ ), 6.47 (ddd, $J=7.0,7.0,1.0,1 \mathrm{H}), 9.38(\mathrm{~s}, 1 \mathrm{H}) ;{ }^{13} \mathrm{C}$ NMR $\left(100 \mathrm{MHz}, \mathrm{CDCl}_{3}\right) \delta-4.9,-4.8,-4.7$, $4.4,-4.3,-3.8,4.8,9.2,11.6,17.9,18.1,18.2,25.7,25.8,25.9,29.4,34.5,35.1,38.5$, $39.6,48.7,58.4,58.8,69.0,69.7,70.1,73.4,75.5,77.5,79.3,80.6,100.0,139.6,153.8$, $154.0,195.0$; IR (thin film) $\vee 2954(\mathrm{~s}), 2929(\mathrm{~s}), 2888(\mathrm{~s}), 2857(\mathrm{~s}), 1815(\mathrm{~s}), 1690(\mathrm{~s})$,

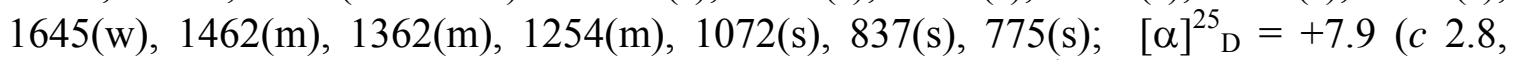
$\mathrm{CH}_{2} \mathrm{Cl}_{2}$ ); MS (ESI) calculated for $\mathrm{C}_{43} \mathrm{H}_{84} \mathrm{NaO}_{11} \mathrm{Si}_{3}[\mathrm{M}+\mathrm{Na}]^{+}: \mathrm{m} / \mathrm{z}$ 883.5, found: $\mathrm{m} / \mathrm{z}$ 883.6.
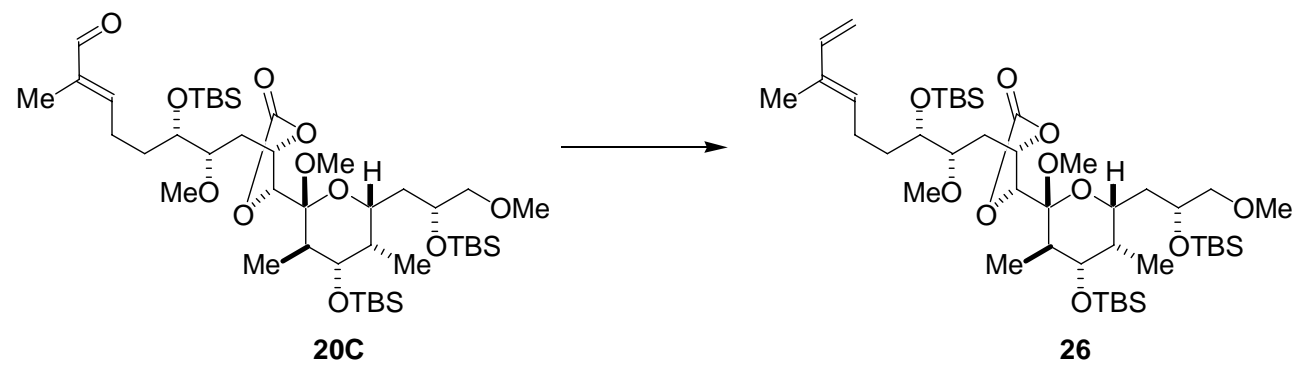

Diene 26. A stirring suspension of methyltriphenylphosphonium bromide $(0.357 \mathrm{~g}, 1.00$ $\mathrm{mmol})$ in THF $(1 \mathrm{~mL})$ was cooled in an ice bath. A solution of $\mathrm{KOt}-\mathrm{Bu}(0.112 \mathrm{mg}, 1.00$ mmol) in THF $(1 \mathrm{~mL})$ was added, dropwise. The yellow methylenetriphenylphosphorane solution was stirred at $\mathrm{rt}$ for $0.5 \mathrm{~h}$. Methylenetriphenylphosphorane solution was added dropwise to a stirring solution of aldehyde 20C, in THF $(2.0 \mathrm{~mL})$, until a bright yellow color persisted then the reaction mixture was diluted with saturated $\mathrm{NH}_{4} \mathrm{Cl}$ solution and $\mathrm{CH}_{2} \mathrm{Cl}_{2}$. The mixture was shaken and separated. The aqueous solution was extracted with $\mathrm{CH}_{2} \mathrm{Cl}_{2}(2 \mathrm{x})$ and the combined organic solution was dried $\left(\mathrm{Na}_{2} \mathrm{SO}_{4}\right)$ and evaporated. Chromatography, eluting with 6:94 EtOAc/hexanes, afforded $0.232 \mathrm{~g}(98 \%)$ of diene 26 as a colorless oil. ${ }^{1} \mathrm{H}$ NMR $\left(400 \mathrm{MHz}, \mathrm{CDCl}_{3}\right) \delta 0.03(\mathrm{~s}, 3 \mathrm{H}), 0.06(\mathrm{~s}, 9 \mathrm{H}), 0.08(\mathrm{~s}, 3 \mathrm{H})$, 0.10 (s, 3H), 0.85 (d, $J=7.0,3 \mathrm{H}), 0.88$ (s, 9H), 0.891 (s, 9H), 0.894 (s, 9H), 0.99 (d, $J=$ $6.5,3 \mathrm{H}), 1.31(\mathrm{~m}, 1 \mathrm{H}), 1.47$ (ddd, $J=14.5,8.0,3.0,1 \mathrm{H}), 1.55-1.75$ (band, $4 \mathrm{H}), 1.73$ (s, $3 \mathrm{H}), 1.88-2.01$ (band, $2 \mathrm{H}), 2.07(\mathrm{~m}, 1 \mathrm{H}), 2.32(\mathrm{~m}, 1 \mathrm{H}), 3.21-3.31$ (band, $2 \mathrm{H}), 3.25(\mathrm{~s}$, $3 \mathrm{H}), 3.30(\mathrm{~s}, 3 \mathrm{H}), 3.38(\mathrm{~m}, 1 \mathrm{H}), 3.39(\mathrm{~s}, 3 \mathrm{H}), 3.71(\mathrm{dd}, J=10.5,5.0,1 \mathrm{H}), 3.84-3.93$ (band, $3 \mathrm{H}), 4.35(\mathrm{~d}, J=5.0,1 \mathrm{H}), 4.88(\mathrm{ddd}, J=10.5,5.0,3.0,1 \mathrm{H}), 4.92(\mathrm{~d}, J=10.5$, $1 \mathrm{H}), 5.08(\mathrm{~d}, J=17.0,1 \mathrm{H}), 5.47$ (br. $\mathrm{t}, J=7.0,1 \mathrm{H}), 6.34(\mathrm{dd}, J=17.0,11.0,1 \mathrm{H}) ;{ }^{13} \mathrm{C}$ NMR $\left(100 \mathrm{MHz}, \mathrm{CDCl}_{3}\right) \delta-4.8,-4.75,-4.65,-4.37,-4.35,-3.8,4.8,11.7,18.0,18.1$, 18.2, 25.0, 25.8, 25.9, 30.5, 34.6, 35.1, 38.5, 39.6, 48.7, 58.2, 58.7, 69.0, 69.7, 70.2, 73.4, 75.6, 77.6, 79.4, 80.6, 100.1, 110.7, 132.5, 134.3, 141.3, 153.9; IR (thin film) v 2954(s), 
2929(s), 2888(s), 2857(s), 1818(s), 1462(m), 1362(m), 1254(s), 1074(s), 836(s), 775(s); $[\alpha]_{D}^{27}=+6.6\left(\right.$ c 3.2, $\mathrm{CH}_{2} \mathrm{Cl}_{2}$ ); MS (ESI) calculated for $\mathrm{C}_{44} \mathrm{H}_{86} \mathrm{NaO}_{10} \mathrm{Si}_{3} \quad[\mathrm{M}+\mathrm{Na}]^{+}: \mathrm{m} / \mathrm{z}$ 881.5 , found: $\mathrm{m} / \mathrm{z} 881.7$.

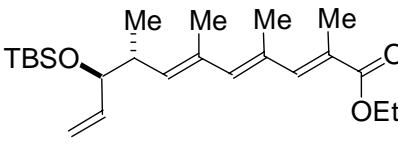

3-TBS

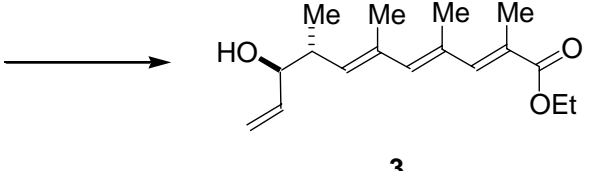

3

Tetraene 3. To a stirring solution of tetraene 3-TBS $(0.583 \mathrm{~g}, 1.49 \mathrm{mmol})$ in $\mathrm{MeCN}$ $(5.00 \mathrm{~mL})$ was added a solution $(20-25 \mathrm{wt} \%)$ of $\mathrm{H}_{2} \mathrm{SiF}_{6}(0.40 \mathrm{~mL})$. After $5 \mathrm{~h}$ the reaction mixture was diluted with $\mathrm{NaHCO}_{3}$ solution, then extracted with $\mathrm{CH}_{2} \mathrm{Cl}_{2}$ (3x). The combined organic solution was dried $\left(\mathrm{Na}_{2} \mathrm{SO}_{4}\right)$ and evaporated. Chromatography, eluting with 16:84 EtOAc/hexanes, afforded $0.399 \mathrm{~g} \mathrm{(96 \% )}$ of tetraene 3 as a colorless oil. ${ }^{1} \mathrm{H}$ NMR $\left(400 \mathrm{MHz}, \mathrm{CDCl}_{3}\right) \delta 1.02(\mathrm{~d}, J=7.0,3 \mathrm{H}), 1.27(\mathrm{t}, J=7.0,3 \mathrm{H}), 1.77(\mathrm{~d}, J=1.0$, $3 \mathrm{H}), 1.92(\mathrm{~m}, 1 \mathrm{H}), 1.95(\mathrm{~d}, J=1.0,3 \mathrm{H}), 2.00(\mathrm{~d}, J=1.0,3 \mathrm{H}), 2.64$ (ddddd, $J=10.0$, $4 \times 6.5,1 \mathrm{H}), 3.97(\mathrm{~m}, 1 \mathrm{H}), 4.17(\mathrm{q}, J=7.0,2 \mathrm{H}), 5.13(\mathrm{dt}, J=9.0,1.0,1 \mathrm{H}), 5.23(\mathrm{dt}, J=$ 17.0, 1.0, 1H), 5.24 (br. d, $J=10.0,1 \mathrm{H}), 5.87$ (ddd, $J=17.0,10.5,6.0,1 \mathrm{H}), 5.98(\mathrm{~s}, 1 \mathrm{H})$, $7.12(\mathrm{~s}, 1 \mathrm{H}) ;{ }^{13} \mathrm{C} \mathrm{NMR}\left(100 \mathrm{MHz}, \mathrm{CDCl}_{3}\right) \delta 14.0,14.2,16.4,17.2,18.3,39.0,60.5,76.7$, 115.4, 125.8, 131.8, 133.0, 133.5, 138.6, 139.0, 143.6, 169.1; IR (thin film) $\vee 3476(\mathrm{br})$, 2979(m), 2929(m), 1702(s), 1611(m), 1446(m), 1367(m), 1255(s), 1114(s), 1029(m), 988(m), 922(m), 751(m); $[\alpha]^{23}=+109$ (c 3.6, $\left.\mathrm{CH}_{2} \mathrm{Cl}_{2}\right)$; MS (ESI) calculated for $\mathrm{C}_{17} \mathrm{H}_{26} \mathrm{NaO}_{3}[\mathrm{MNa}]^{+}: \mathrm{m} / \mathrm{z}$ 301.2, found: $\mathrm{m} / \mathrm{z} 301.4$.

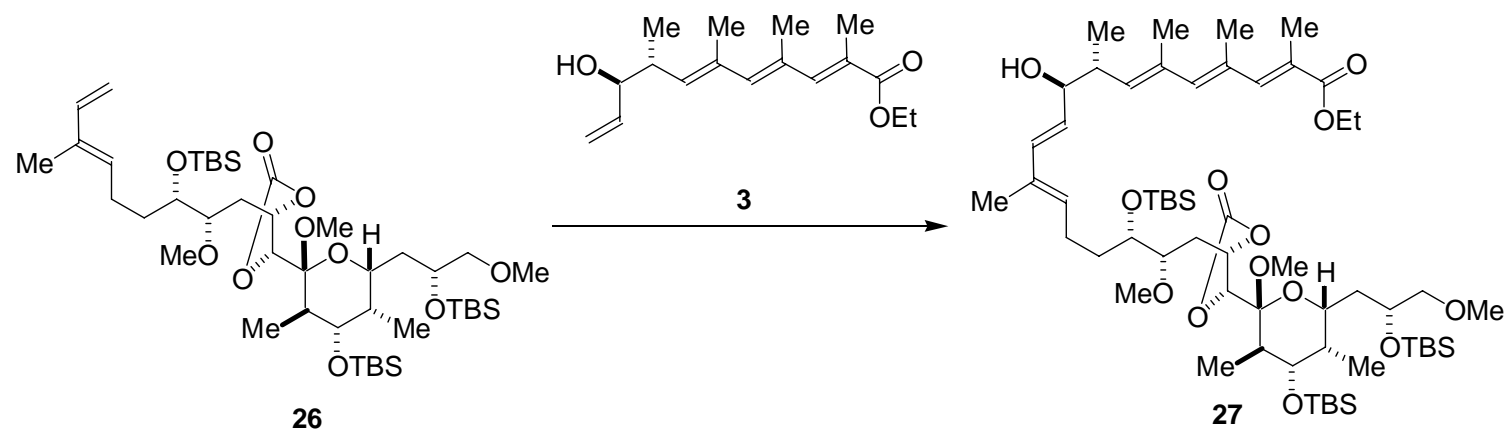

Pentaene 27. To a solution of diene $26(23.4 \mathrm{mg}, 0.0272 \mathrm{mmol})$ and tetraene $3(17.5 \mathrm{mg}$, $0.0628 \mathrm{mmol})$ in $\mathrm{CH}_{2} \mathrm{Cl}_{2}(0.30 \mathrm{~mL})$ was added Grubbs second generation catalyst ${ }^{2}$ $\left(\mathrm{Cl}_{2}\left(\mathrm{PCy}_{3}\right)(\mathrm{IMes}) \mathrm{Ru}=\mathrm{CHPh}\right)(2.7 \mathrm{mg}, 0.0031 \mathrm{mmol})$. The homogeneous solution was allowed to stand, under an argon atmosphere, at $\mathrm{rt}$ for $3 \mathrm{~h}$. The solution was then stirred in the air for $2 \mathrm{~h}$ before being evaporated. Chromatography, eluting with 1:3 EtOAc/hexanes, ${ }^{*}$ afforded $7.3 \mathrm{mg}$ (31\%) of recovered diene 26 and $19.0 \mathrm{mg}(63 \%)$ of pentaene 27 as colorless oils. An analytical sample of pentaene 27 was obtained after HPLC purification (Gradient elution $10 \rightarrow 15 \%$ EtOAc in hexanes), the major impurity 
was not an isomer of 27. $* \mathrm{Rf} \sim 0.5$, at lower $\mathrm{Rf}(0.3)$ the product coelutes with tetraene 3 . ${ }^{1} \mathrm{H} \mathrm{NMR}\left(500 \mathrm{MHz}, \mathrm{CDCl}_{3}\right) \delta 0.03$ (s, 3H), 0.06 (band, 9H), 0.07 (s, 3H), $0.09(\mathrm{~s}, 3 \mathrm{H})$, $0.85(\mathrm{~d}, J=7.0,3 \mathrm{H}), 0.88(\mathrm{~s}, 9 \mathrm{H}), 0.89$ (band, $18 \mathrm{H}), 0.98(\mathrm{~d}, J=7.0,3 \mathrm{H}), 1.05$ (d, $J=$ $6.5,3 \mathrm{H}), 1.25-1.35(\mathrm{~m}, 1 \mathrm{H}), 1.30(\mathrm{t}, J=7.0,3 \mathrm{H}), 1.48(\mathrm{ddd}, J=14.5,8.0,3.5,1 \mathrm{H}), 1.56-$ 1.72 (band, 4H), $1.72(\mathrm{~s}, 3 \mathrm{H}), 1.80(\mathrm{~s}, 3 \mathrm{H}), 1.90-2.01$ (band, 2H), 1.97 (s, 3H), 2.02 (s, $3 \mathrm{H}), 2.06(\mathrm{~m}, 1 \mathrm{H}), 2.31(\mathrm{~m}, 1 \mathrm{H}), 2.69(\mathrm{~m}, 1 \mathrm{H}), 3.22-3.32$ (band, $2 \mathrm{H}), 3.24(\mathrm{~s}, 3 \mathrm{H}), 3.30$ $(\mathrm{s}, 3 \mathrm{H}), 3.38(\mathrm{~m}, 1 \mathrm{H}), 3.40(\mathrm{~s}, 3 \mathrm{H}), 3.71(\mathrm{dd}, J=10.5,4.5,1 \mathrm{H}), 3.83-3.92$ (band, $3 \mathrm{H})$, 4.05 (br. ddd, $J=6.0,6.0,5.0,1 \mathrm{H}), 4.20$ (q, $J=7.0,2 \mathrm{H}), 4.35$ (d, $J=5.0,1 \mathrm{H}), 4.89$ (m, $1 \mathrm{H}), 5.27$ (d, $J=10.0,1 \mathrm{H}), 5.46$ (br. t, $J=7.0,1 \mathrm{H}), 5.59$ (dd, $J=15.5,6.5,1 \mathrm{H}), 6.02$ (s, $1 \mathrm{H}), 6.22(\mathrm{~d}, J=15.5,1 \mathrm{H}), 7.15(\mathrm{~s}, 1 \mathrm{H}) ;{ }^{13} \mathrm{C} \mathrm{NMR}\left(125 \mathrm{MHz}, \mathrm{CDCl}_{3}\right) \delta-4.8,-4.72$, 4.67, -4.34, -4.32, -3.8, 4.9, 11.7, 12.5, 14.1, 14.3, 16.6, 17.3, 18.0, 18.1, 18.2, 18.3, 25.0, $25.77,25.81,25.9,30.5,34.6,35.1,38.5,39.4,39.6,48.7,58.3,58.8,60.6,69.0,69.7$, 70.3, 73.4, 75.6, 77.6, 79.3, 80.7, 100.1, 125.9, 127.2, 131.9, 132.7, 133.1, 133.3, 133.7, 136.2, 138.8, 143.6, 154.0, 169.1; IR (thin film) v: 3510(br.), 2929(s), 2857(s), 1816(s), 1704(s), 1613(w), 1463(m), 1367(m), 1254(s), 1110(s), 837(s), 775(s); $[\alpha]^{27}{ }_{\mathrm{D}}=+46(\mathrm{c}$ $0.21, \mathrm{CH}_{2} \mathrm{Cl}_{2}$ ); MS (ESI) calculated for $\mathrm{C}_{59} \mathrm{H}_{108} \mathrm{NaO}_{13} \mathrm{Si}_{3}[\mathrm{M}+\mathrm{Na}]^{+}: \mathrm{m} / \mathrm{z} 1131.7$, found: $\mathrm{m} / \mathrm{z} 1131.9$.
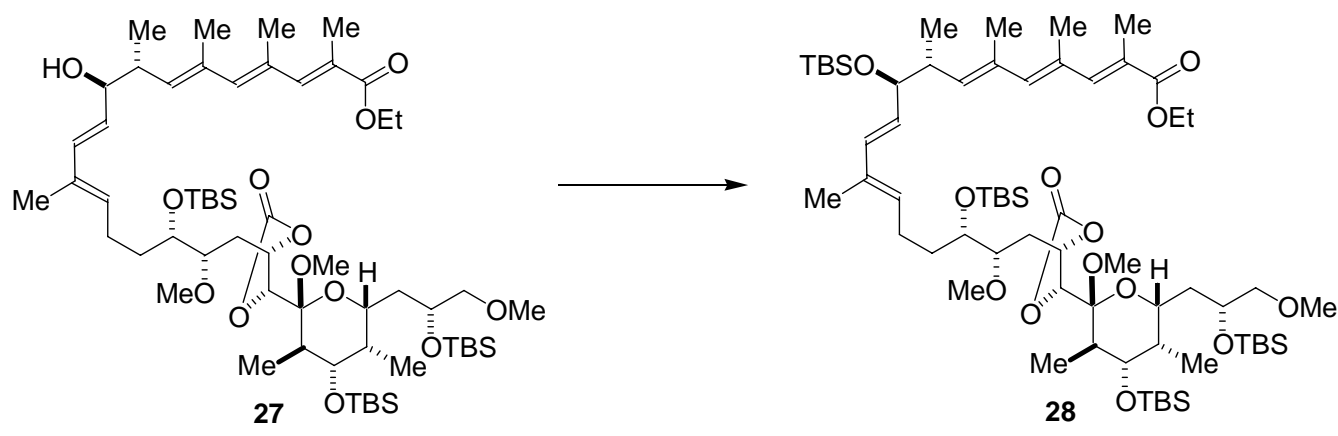

TBS ether 28. A solution of pentaene $27(68.5 \mathrm{mg}, 0.0617 \mathrm{mmol})$, tertbutyldimethylsilyl chloride (52.0 $\mathrm{mg}, 0.345 \mathrm{mmol})$, and imidazole (105 $\mathrm{mg}, 1.54 \mathrm{mmol})$ in DMF $(0.50 \mathrm{~mL})$ was allowed to stand at $\mathrm{rt}$ for $15 \mathrm{~h}$. The solution was diluted with $\mathrm{H}_{2} \mathrm{O}$ then extracted with EtOAc $(3 \mathrm{x})$. The combined organic solution was dried $\left(\mathrm{Na}_{2} \mathrm{SO}_{4}\right)$ then evaporated. Chromatography, eluting with 7:93 EtOAc/hexanes, afforded $56.9 \mathrm{mg}$ (75\%) of silyl ether 28 as a colorless oil. ${ }^{1} \mathrm{H}$ NMR $\left(500 \mathrm{MHz}, \mathrm{CDCl}_{3}\right) \delta-0.03(\mathrm{~s}, 3 \mathrm{H})$, $0.013(\mathrm{~s}, 3 \mathrm{H}), 0.018(\mathrm{~s}, 3 \mathrm{H}), 0.04(\mathrm{~s}, 3 \mathrm{H}), 0.05$ (band, 6H), $0.06(\mathrm{~s}, 3 \mathrm{H}), 0.08(\mathrm{~s}, 3 \mathrm{H}), 0.84$ $(\mathrm{d}, J=7.0,3 \mathrm{H}), 0.86(\mathrm{~s}, 9 \mathrm{H}), 0.88($ band, $27 \mathrm{H}), 0.95-1.01(\mathrm{~m}, 1 \mathrm{H}), 0.975(\mathrm{~d}, J=6.5,3 \mathrm{H})$, $0.977(\mathrm{~d}, J=7.0,3 \mathrm{H}), 1.22-1.35(\mathrm{~m}, 1 \mathrm{H}), 1.28(\mathrm{t}, J=7.0,3 \mathrm{H}), 1.47$ (ddd, $J=14.5,8.0$, 3.5, 1H), 1.55-1.71 (band, 3H), $1.69(\mathrm{~s}, 3 \mathrm{H}), 1.73(\mathrm{~s}, 3 \mathrm{H}), 1.90-1.99$ (band, $2 \mathrm{H}), 1.95$ (s, $3 \mathrm{H}), 2.00(\mathrm{~s}, 3 \mathrm{H}), 2.07(\mathrm{~m}, 1 \mathrm{H}), 2.29(\mathrm{~m}, 1 \mathrm{H}), 2.55(\mathrm{~m}, 1 \mathrm{H}), 3.22-3.30$ (band, $2 \mathrm{H}), 3.24$ $(\mathrm{s}, 3 \mathrm{H}), 3.29(\mathrm{~s}, 3 \mathrm{H}), 3.37(\mathrm{~m}, 1 \mathrm{H}), 3.38(\mathrm{~s}, 3 \mathrm{H}), 3.70(\mathrm{dd}, J=10.5,5.0,1 \mathrm{H}), 3.84-3.91$ (band, 3H), 3.97 (t, $J=6.0,1 \mathrm{H}), 4.18(\mathrm{q}, J=7.0,1 \mathrm{H}), 4.35(\mathrm{~d}, J=4.5,1 \mathrm{H}), 4.88(\mathrm{~m}, 1 \mathrm{H})$, $5.24(\mathrm{~d}, J=10.0,1 \mathrm{H}), 5.38(\mathrm{t}, J=6.5,1 \mathrm{H}), 5.50(\mathrm{dd}, J=16.0,7.0,1 \mathrm{H}), 5.98(\mathrm{~s}, 1 \mathrm{H}), 6.09$ $(\mathrm{d}, J=16.0,1 \mathrm{H}), 7.14(\mathrm{~s}, 1 \mathrm{H}) ;{ }^{13} \mathrm{C}$ NMR $\left(125 \mathrm{MHz}, \mathrm{CDCl}_{3}\right) \delta-4.89,-4.88,-4.8,-4.7,-$ 
$4.4,-4.1,-3.8,4.8,11.6,12.5,14.1,14.3,16.3,17.2,18.16,18.17,18.20,24.9,25.72$, $25.76,25.84,25.88,30.4,34.5,35.0,38.5,39.6,40.4,48.7,58.1,58.7,60.5,69.0,69.7$, 70.0, 73.4, 75.6, 77.5, 77.7, 79.3, 80.5, 100.0, 125.5, 128.6, 131.4, 131.50, 131.52, 133.4, $134.9,135.1,139.3,143.8,153.9,169.0 ;$ IR (thin film) v: 2954(s), 2929(s), 2857(s), 1818(s), 1705(s), 1463(m), 1365(m), 1253(s), 1111(s), 836(s), 775(s); $[\alpha]_{\mathrm{D}}^{24}=+49(\mathrm{c}$ 2.9, $\mathrm{CH}_{2} \mathrm{Cl}_{2}$ ); MS (ESI) calculated for $\mathrm{C}_{65} \mathrm{H}_{122} \mathrm{NaO}_{13} \mathrm{Si}_{4}[\mathrm{M}+\mathrm{Na}]^{+}: \mathrm{m} / \mathrm{z} 1245.8$, found: $\mathrm{m} / \mathrm{z} 1246.9$.
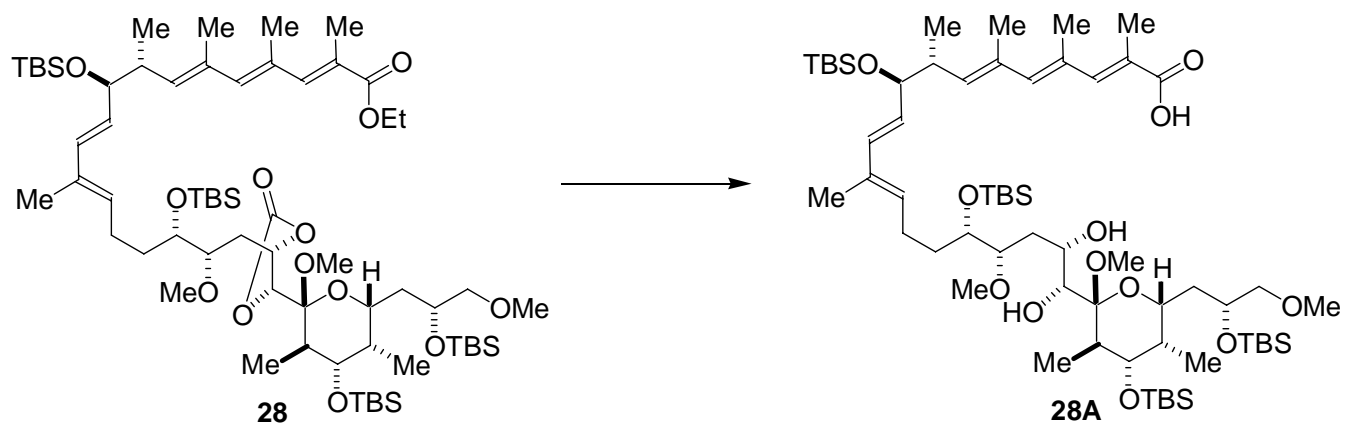

Acid 28A. To a solution of ester $28(56.9 \mathrm{mg}, 0.0475 \mathrm{mmol})$ in a mixture of THF $(0.60$ $\mathrm{mL}), \mathrm{MeOH}(0.20 \mathrm{~mL})$, and $\mathrm{H}_{2} \mathrm{O}(0.10 \mathrm{~mL})$ was added $\mathrm{LiOH} \cdot \mathrm{H}_{2} \mathrm{O}(20 \mathrm{mg}, 0.48 \mathrm{mmol})$. The cloudy solution was stirred vigorously for $2.5 \mathrm{~d}$ before being diluted with $\mathrm{NH}_{4} \mathrm{Cl}$ solution and then extracted with EtOAc $(5 \mathrm{x})$. The combined organic solution was dried $\left(\mathrm{Na}_{2} \mathrm{SO}_{4}\right)$ and evaporated. Chromatography, eluting with 17:83 then 1:4 then 1:3 EtOAc/hexanes afforded $42.7 \mathrm{mg}(77 \%)$ of acid $\mathbf{2 8 A}$ as a white amorphous solid. ${ }^{1} \mathrm{H}$ NMR $\left(500 \mathrm{MHz}, \mathrm{CDCl}_{3}\right) \delta-0.01(\mathrm{~s}, 3 \mathrm{H}), 0.02(\mathrm{~s}, 3 \mathrm{H}), 0.03(\mathrm{~s}, 3 \mathrm{H}), 0.04(\mathrm{~s}, 3 \mathrm{H}), 0.07$ (band, 6H), $0.08(\mathrm{~s}, 3 \mathrm{H}), 0.10(\mathrm{~s}, 3 \mathrm{H}), 0.86(\mathrm{~d}, J=6.5,3 \mathrm{H}), 0.88$ (band, $18 \mathrm{H}), 0.89$ (s, $9 \mathrm{H}), 0.90(\mathrm{~s}, 9 \mathrm{H}), 0.99(\mathrm{~d}, J=6.5,3 \mathrm{H}), 1.00(\mathrm{~d}, J=6.5,3 \mathrm{H}), 1.39(\mathrm{~m}, 1 \mathrm{H}), 1.46(\mathrm{~m}, 1 \mathrm{H})$, $1.52(\mathrm{~m}, 1 \mathrm{H}), 1.60(\mathrm{~m}, 1 \mathrm{H}), 1.64-1.78$ (band, $2 \mathrm{H}), 1.70(\mathrm{~s}, 3 \mathrm{H}), 1.75(\mathrm{~s}, 3 \mathrm{H}), 1.90(\mathrm{~m}$, $1 \mathrm{H}), 1.98(\mathrm{~s}, 3 \mathrm{H}), 2.03(\mathrm{~s}, 3 \mathrm{H}), 2.04-2.13$ (band, $2 \mathrm{H}), 2.29(\mathrm{~m}, 1 \mathrm{H}), 2.57(\mathrm{~m}, 1 \mathrm{H}), 3.26(\mathrm{~s}$, $3 \mathrm{H}), 3.27-3.34$ (band, $2 \mathrm{H}), 3.33(\mathrm{~s}, 3 \mathrm{H}), 3.41(\mathrm{~s}, 3 \mathrm{H}), 3.47(\mathrm{~m}, 1 \mathrm{H}), 3.49(\mathrm{~s}, 1 \mathrm{H}), 3.72$ (dd, $J=10.5,4.5,1 \mathrm{H}$ ), 3.81-3.93 (band, 3H), 3.98 (br. t, $J=6.5,1 \mathrm{H}$ ), 4.02 (br. d, $J=8.5$, $1 \mathrm{H}), 5.28$ (br. d, $J=9.5,1 \mathrm{H}$ ), 5.40 (br. t, $J=7.0,1 \mathrm{H}$ ), 5.50 (dd, $J=15.5,7.0,1 \mathrm{H}$ ), 6.05 $(\mathrm{s}, 1 \mathrm{H}), 6.94(\mathrm{~d}, J=15.5,1 \mathrm{H}), 7.27(\mathrm{~s}, 1 \mathrm{H}) ;{ }^{13} \mathrm{C} \mathrm{NMR}\left(125 \mathrm{MHz}, \mathrm{CDCl}_{3}\right) \delta-4.83,-4.81$, $-4.69,-4.67,-4.33,-4.32,-4.0,-3.9,5.2,12.2,12.5,13.8,16.4,17.2,18.02,18.08,18.13$, $18.21,18.23,25.0,25.836,25.842,25.90,25.93,31.2,34.9,35.9,38.7,39.7,40.5,48.4$, 58.2, 58.8, 67.1, 68.9, 69.9, 71.4, 73.4, 74.8, 77.5, 77.9, 81.1, 102.6, 124.4, 128.4, 131.4, 131.5, 132.0, 133.2, 135.2, 135.7, 140.7, 146.2, 174.0; IR (thin film) v 3445(br), 2954(s), 2929(s), 2892(s), 2857(s), 1682(s), 1471(m), 1463(m), 1255(s), 1070 (s), 836(s), 775(s); $[\alpha]_{D}^{23}=+33\left(\right.$ c $\left.1.5, \mathrm{CH}_{2} \mathrm{Cl}_{2}\right)$; MS (ESI) calculated for $\mathrm{C}_{62} \mathrm{H}_{120} \mathrm{NaO}_{12} \mathrm{Si}_{4}[\mathrm{M}+\mathrm{Na}]^{+}: \mathrm{m} / \mathrm{z}$ 1191.8, found: $\mathrm{m} / \mathrm{z} 1192.0$. 

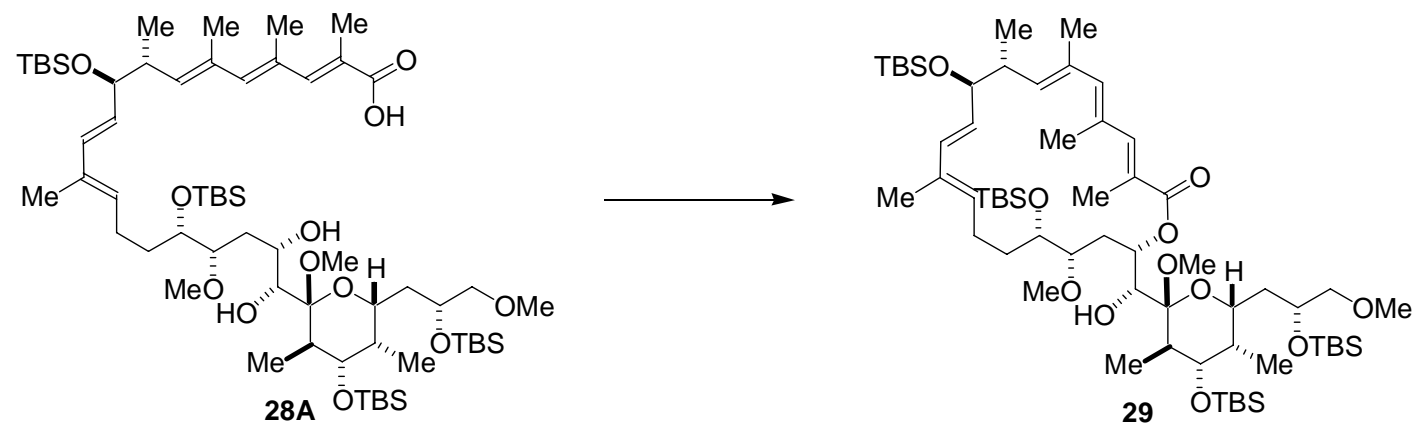

Lactone 29. To a THF solution $(0.40 \mathrm{~mL})$ of acid 28A $(17.5 \mathrm{mg}, 0.0149 \mathrm{mmol})$ and $\mathrm{Et}_{3} \mathrm{~N}(0.062 \mathrm{~mL}, 0.45 \mathrm{mmol})$ was added 2,4,6-trichlorobenzoyl chloride $(0.047 \mathrm{~mL}, 0.30$ $\mathrm{mmol}$ ), dropwise over $5 \mathrm{~min}$. The cloudy solution was stirred at $\mathrm{rt}$ for $4 \mathrm{~h}$. The reaction mixture was diluted with toluene $(0.60 \mathrm{~mL})$ and the resulting cloudy solution added, dropwise over $1 \mathrm{~h}$ (followed by two toluene rinses $(1 \mathrm{~mL})$ ), to a stirring solution of DMAP (100 mg, $0.819 \mathrm{mmol})$ in toluene $(60 \mathrm{~mL})$ at $\mathrm{rt}$. After $20 \mathrm{~h}, \mathrm{NH}_{4} \mathrm{Cl}$ solution and EtOAc were added and the mixture shaken then separated. The aqueous solution was extracted with EtOAc (3x), the combined organic solution was dried $\left(\mathrm{Na}_{2} \mathrm{SO}_{4}\right)$ and evaporated. Chromatography, eluting with 1:9 EtOAc/hexanes, afforded $11.7 \mathrm{mg}(68 \%)$ of lactone 29 as a white, amorphous solid. ${ }^{1} \mathrm{H}$ NMR $\left(500 \mathrm{MHz}, \mathrm{C}_{6} \mathrm{D}_{6}\right) \delta 0.06$ (band, $\left.6 \mathrm{H}\right)$, 0.11 (s, 3H), 0.16(band, 6H), 0.17 (s, 3H), 0.18 (s, 3H), 0.23 (s, 3H), 0.975 (s, 9H), 0.978 (s, 9H), 1.00-1.40 (band, 2H), 1.02 (s, 9H), 1.06 (s, 9H), 1.20 (d, $J=6.5,3 \mathrm{H}), 1.28$ (d, $J=$ 6.5, $3 \mathrm{H}), 1.25-1.36$ (band, $2 \mathrm{H}), 1.48(\mathrm{~d}, J=6.5,3 \mathrm{H}), 1.55-1.67$ (band, $1 \mathrm{H}), 1.58(\mathrm{~s}, 3 \mathrm{H})$, $1.64(\mathrm{~s}, 3 \mathrm{H}), 1.84(\mathrm{~s}, 3 \mathrm{H}), 1.87(\mathrm{~m}, 1 \mathrm{H}), 1.92(\mathrm{~m}, 1 \mathrm{H}), 2.00-2.08(\mathrm{~m}, 1 \mathrm{H}), 2.13(\mathrm{~s}, 3 \mathrm{H})$, $2.17(\mathrm{~m}, 1 \mathrm{H}), 2.26$ (m, 1H), 2.39 (br. d, $J=5.0,1 \mathrm{H}), 2.52-2.62$ (band, $2 \mathrm{H}$ ), 2.98 (t, $J=$ 9.0, $1 \mathrm{H}$ ), 3.05 (s, 3H), 3.12-3.18 (band, $2 \mathrm{H}), 3.28$ (s, 3H), 3.52 (td, $J=9.0,2.0,1 \mathrm{H}), 3.57$ (s, 3H), 3.84 (t, $J=8.5,1 \mathrm{H}), 3.89$ (t, $J=4.5,1 \mathrm{H}), 4.09$ (dd, $J=10.0,5.0,1 \mathrm{H}), 4.12$ (m, $1 \mathrm{H}), 4.19(\mathrm{dt}, J=7.5,2.5,1 \mathrm{H}), 5.02(\mathrm{~d}, J=10.5,1 \mathrm{H}), 5.34(\mathrm{dd}, J=15.5,8.5,1 \mathrm{H}), 5.43$ (br. t, $J=8.0,1 \mathrm{H}), 5.98(\mathrm{~d}, J=15.5,1 \mathrm{H}), 6.00(\mathrm{~m}, 1 \mathrm{H}), 6.15(\mathrm{~s}, 1 \mathrm{H}), 7.49(\mathrm{~s}, 1 \mathrm{H}) ;{ }^{13} \mathrm{C}$ NMR (125 MHz, $\left.\mathrm{C}_{6} \mathrm{D}_{6}\right) \delta-4.7,-4.53,-4.47,-4.2,-4.1,-3.7,-3.48,-3.43,5.8,11.9,12.0$, $14.4,16.5,17.8,18.2,18.40,18.49,18.50,18.6,25.6,26.14,26.21,26.32,26.39,36.3$, 37.1, 38.9, 39.2, 40.6, 41.1, 47.9, 58.5, 61.3, 69.7, 70.6, 71.0, 73.6, 75.9, 76.8, 78.1, 81.7, 82.2, 102.3, 124.6, 129.1, 131.8, 132.4, 132.6, 133.1, 136.8, 141.0, 144.4, 145.1, 169.3; IR (thin film) v: 2955(s), 2928(s), 2857(s), 1701(s), 1462(s), 1388(m), 1361(m), 1251(s), 1110(s), 1073(s), 836(s), 774(s); $[\alpha]^{28}=+13\left(c 0.56, \mathrm{CH}_{2} \mathrm{Cl}_{2}\right)$; MS (ESI) calculated for $\mathrm{C}_{62} \mathrm{H}_{118} \mathrm{NaO}_{11} \mathrm{Si}_{4}[\mathrm{M}+\mathrm{Na}]^{+}: \mathrm{m} / \mathrm{z}$ 1173.8, found: $\mathrm{m} / \mathrm{z}$ 1174.0. 

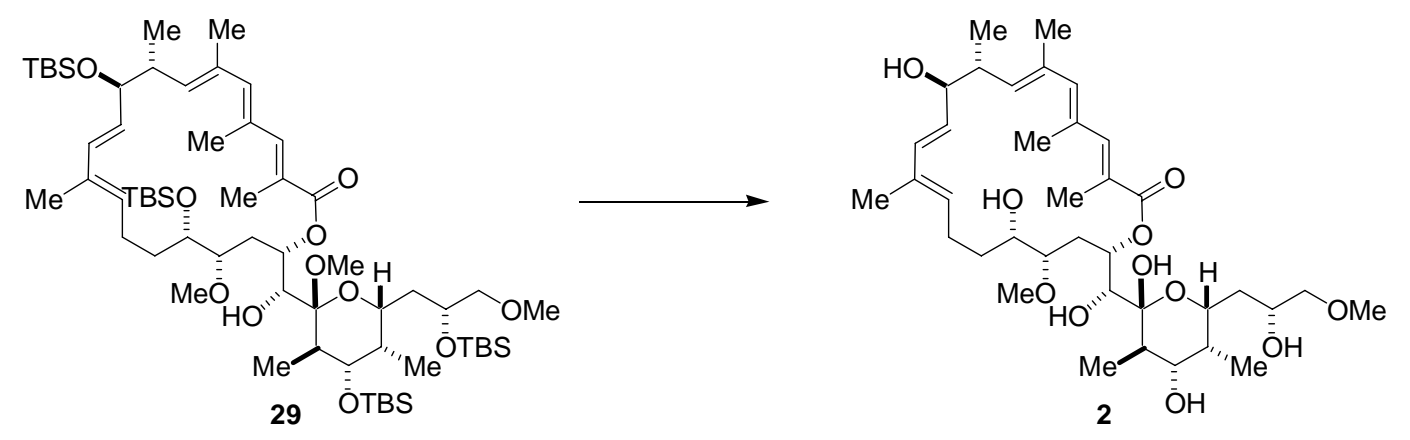

Apoptolidinone (2). A solution of lactone $29(3.3 \mathrm{mg}, 0.0028 \mathrm{mmol})$ in $\mathrm{MeCN}(0.40$ $\mathrm{mL}$ ) was cooled to $-35^{\circ} \mathrm{C}$. An aqueous solution $(20-25 \mathrm{wt} \%)$ of $\mathrm{H}_{2} \mathrm{SiF}_{6}$ (4 drops from a $20 \mathrm{~g}$ needle) was added to the reaction mixture. The mixture was swirled the allowed to stand at $-18{ }^{\circ} \mathrm{C}$ for $2 \mathrm{~d}$ then at $0{ }^{\circ} \mathrm{C}$ for $2 \mathrm{~d}$. After dilution with $\mathrm{NaHCO}_{3}$ solution the mixture extracted with EtOAc $(5 x)$. The combined organic solution was dried $\left(\mathrm{Na}_{2} \mathrm{SO}_{4}\right)$ and evaporated. Chromatography, eluting with 1:9 $\mathrm{MeOH} / \mathrm{CH}_{2} \mathrm{Cl}_{2}$, afforded $1.2 \mathrm{mg}$ (61\%) of apoptolidinone (2) as a white amorphous solid. ${ }^{1} \mathrm{H} \mathrm{NMR}\left(500 \mathrm{MHz}, \mathrm{CD}_{3} \mathrm{OD}\right) \delta$ $0.88(\mathrm{~d}, J=7.0,3 \mathrm{H}), 1.02(\mathrm{~d}, J=6.5,3 \mathrm{H}), 1.13(\mathrm{~d}, J=6.5,3 \mathrm{H}), 1.24-1.36(\mathrm{~m}, 1 \mathrm{H}), 1.41$ (m, 1H), 1.51-1.62 (band, 2H), $1.68(\mathrm{~s}, 3 \mathrm{H}), 1.69-1.80$ (band, $2 \mathrm{H}), 1.93(\mathrm{~s}, 3 \mathrm{H}), 2.00-2.11$ (band, 2H), 2.12 (s, 3H), 2.13-2.18 (m, 1H), 2.19 (s, 3H), 2.40-2.53 (band, 2H), 2.73 (dd, $J=10.0,4.0,1 \mathrm{H}), 3.17(\mathrm{dd}, J=9.0,6.0,1 \mathrm{H}), 3.21(\mathrm{dd}, J=9.5,4.5,1 \mathrm{H}), 3.30(\mathrm{~s}, 3 \mathrm{H})$, $3.36(\mathrm{~s}, 3 \mathrm{H}), 3.44(\mathrm{~m}, 1 \mathrm{H}), 3.52-3.58$ (band, $2 \mathrm{H}), 3.72-3.80$ (band, $2 \mathrm{H}), 4.09$ (dm, $J=9.0$, $1 \mathrm{H}), 5.22(\mathrm{~d}, J=10.0,1 \mathrm{H}), 5.30(\mathrm{~d}, J=11.5,1 \mathrm{H}), 5.33(\mathrm{dd}, J=15.5,9.0,1 \mathrm{H}), 5.65(\mathrm{dd}$, $J=9.0,7.0,1 \mathrm{H}), 6.10(\mathrm{~d}, J=15.5,1 \mathrm{H}), 6.19(\mathrm{~s}, 1 \mathrm{H}), 7.37(\mathrm{~s}, 1 \mathrm{H}) ;{ }^{13} \mathrm{C}$ NMR $(125 \mathrm{MHz}$, $\left.\mathrm{CD}_{3} \mathrm{OD}\right) \delta 5.3,12.1,12.2,14.0,16.4,17.77,17.82,24.5,36.4,36.6,38.4,38.6,40.8$, 41.0, 59.4, 61.4, 68.1, 69.2, 72.3, 73.7, 74.6, 75.5, 78.6, 80.6, 83.8, 101.3, 123.8, 129.6, $132.6,133.0,133.1,134.9,137.7,143.7,147.3,149.1,172.7 ;[\alpha]^{27}{ }_{\mathrm{D}}=+13(c 0.1$, $\mathrm{CH}_{2} \mathrm{Cl}_{2}$ ); MS (ESI) calculated for $\mathrm{C}_{37} \mathrm{H}_{60} \mathrm{NaO}_{11}[\mathrm{M}+\mathrm{Na}]^{+}: \mathrm{m} / \mathrm{z}$ 703.4, found: $\mathrm{m} / \mathrm{z}$ 703.5.

1. Gottlieb, H. E.; Kotlyar, V.; Nudelman, A. J. Org. Chem. 1997, 62, 7512.

2. Scholl, M.; Ding, S.; Lee, C. W.; Grubbs, R. H. Org. Lett. 1999, 1, 953. 